\begin{tabular}{|c|c|c|c|}
\hline AHMAD MUNEER SHARAYAH, MD & SAMRAH ZAIGHAM, MD & NOOR HAJJAJ, MD & MIHIR MANIAR, DO \\
\hline $\begin{array}{l}\text { Chief Internal Medicine Resident, Monmouth } \\
\text { Medical Center, Long Branch, NJ }\end{array}$ & $\begin{array}{l}\text { Department of Internal Medicine, } \\
\text { Monmouth Medical Center, } \\
\text { Long Branch, NJ }\end{array}$ & $\begin{array}{l}\text { Faculty of Medicine, } \\
\text { University of Jordan, } \\
\text { Amman, Jordan }\end{array}$ & $\begin{array}{l}\text { Department of Internal Medicine, } \\
\text { Monmouth Medical Center, } \\
\text { Long Branch, NJ }\end{array}$ \\
\hline
\end{tabular}

\title{
A complication of enoxaparin injection
}

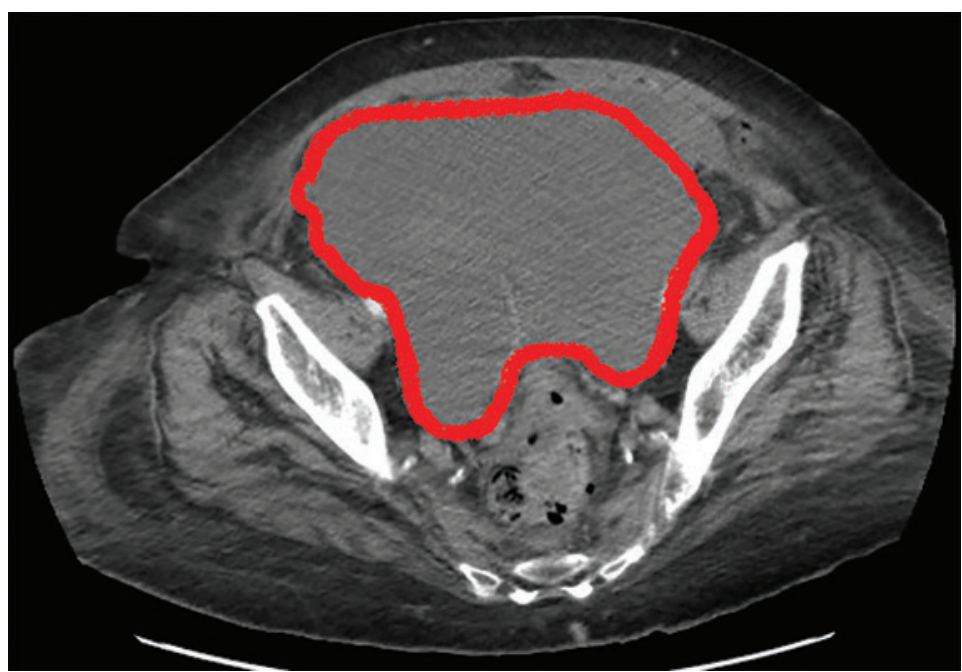

Figure 1. Abdominal computed tomography revealed a hematoma $15 \mathrm{~cm} \times 15 \mathrm{~cm}$ within the paracolic gutter and pelvis, causing bladder compression and left-sided hydronephrosis. of residual urine. She was hemodynamically stable, but physical examination revealed mild abdominal distention and tenderness in the suprapubic region. Laboratory testing showed a sharp rise in serum creatinine and a drop in hematocrit.

Computed tomography of the abdomen revealed a hematoma measuring 15 by $15 \mathrm{~cm}$ within the paracolic gutter and pelvis, compressing the bladder and causing left-sided hydronephrosis (Figure 1). Her laboratory abnormalities were therefore interpreted as postrenal acute kidney injury and anemia due to blood loss.

The patient was initially managed conservatively with serial physical examinations, monitoring of the hematocrit, serial imaging studies, and discontinuation of anticoagulation, but the pain and anuria persisted. Repeat computed tomography 15 days after admission showed that the hematoma had expanded, and she now had hydronephrosis on the right side as well, requiring urologic intervention with bilateral nephrostomy tube placement.

The size of the hematoma was evaluated with serial abdominal and pelvic examinations. After several days, her urine output had improved, the nephrostomy tubes were removed, and she was discharged.

\section{RECTUS SHEATH HEMATOMA}

Our patient had a giant pelvic hematoma, probably arising from the rectus sheath. This uncommon problem can arise from trauma, anticoagulation, or increased intra-abdominal pressure, but it can also occur spontaneously. ${ }^{1}$

In rectus sheath hematoma, a branch of the inferior epigastric artery is injured at its insertion into the rectus abdominis muscle. Symptoms arise if bleeding does not stop 
spontaneously from a tamponade effect. ${ }^{2}$

We speculate that in our patient, deep injection of enoxaparin into the abdominal wall injured the inferior epigastric artery, which started the hematoma, and the bleeding was exacerbated by the anticoagulation effect of the enoxaparin.

Another form of pelvic hematoma is retroperitoneal. It is most commonly caused by trauma but can occur due to rupture of the aorta, compression from tumors, or, infrequently, anticoagulation therapy. ${ }^{3}$

\section{The role of anticoagulation}

Spontaneous pelvic hematoma is usually missed as a cause of abdominal pain in patients on anticoagulation therapy and is mistaken for common acute conditions such as ulcer, diverticulitis, appendicitis, ovarian cyst torsion, and tumor. ${ }^{4}$ It usually develops within 5 days of starting anticoagulation therapy. Symptoms vary depending on the location of the hematoma and are best diagnosed with abdominal computed tomography, with sensitivity as high as $100 \%$.

\section{REFERENCES}

1. Cherry WB, Mueller PS. Rectus sheath hematoma: review of 126 cases at a single institution. Medicine (Baltimore) 2006; 85(2):105-110. doi:10.1097/01.md.0000216818.13067.5a

2. Hatjipetrou A, Anyfantakis D, Kastanakis M. Rectus sheath hematoma: a review of the literature. Int J Surg 2015; 13:267-271. doi:10.1016/j.ijsu.2014.12.015

3. Haq MM, Taimur SDM, Khan SR, Rahman MA. Retroperitoneal hematoma following enoxaparin treatment in an elderly woman-a case report. Cardiovasc J 2010; 3(1):94-97. doi:10.3329/cardio.v3i1.6434

4. Luhmann A, Williams EV. Rectus sheath hematoma: a series of unfortunate events. World J Surg 2006; 30(11):2050-2055. doi:10.1007/s00268-005-0702-9

\section{MANAGEMENT}

Conservative management, reserved for patients in stable condition, includes temporarily stopping and reevaluating the risks and benefits of anticoagulation and antiplatelet agents, giving blood transfusions, and controlling pain. If conservative measures fail, options are arterial embolization, stent grafting, and blood vessel ligation. ${ }^{5}$ If these measures fail, patients should undergo surgical evacuation of the hematoma and ligation of bleeding vessels. ${ }^{6}$

\section{TAKE-HOME MESSAGE}

Subcutaneous injections, especially of anticoagulants, into the abdominal wall can increase the risk of hematoma. Other risk factors are older age, female sex, and thin body habitus with less abdominal fat. ${ }^{7}$ Healthcare professionals should avoid deep injections into the abdomen and should counsel patients and their caregivers about this, as well. The deltoid region could be a safer alternative.

5. Pace F, Colombo GM, Del Vecchio LR, et al. Low molecu lar weight heparin and fatal spontaneous extraperitoneal hematoma in the elderly. Geriatr Gerontol Int 2012; 12(1):172-174. doi:10.1111/j.1447-0594.2011.00742.x

6. Velicki L, Cemerlic-Adic N, Bogdanovic D, Mrdanin T. Rectus sheath haematoma: enoxaparin-related complication. Acta Clin Belg 2013; 68(2):147-149. doi:10.2143/ACB.68.2.3213

7. Sheth HS, Kumar R, DiNella J, Janov C, Kaldas H, Smith RE. Evaluation of risk factors for rectus sheath hematoma. Clin Appl Thromb Hemost 2016; 22(3):292-296. doi:10.1177/1076029614553024

ADDRESS: Ahmad Muneer Sharayah, MD, Department of Internal Medicine, Monmouth Medical Center, 300 2nd Avenue, Long Branch, NJ, 07740; drsharayah@gmail.com 